\title{
On Asymptotics for a Class of Radial Fourier Transforms
}

\author{
E. Liflyand and W. Trebels
}

\begin{abstract}
A connection is established between the multidimensional Fourier transform of a radial function $f$ from a given class and the one-dimensional Fourier transform of a related function. This is applied to give an asymptotic formula for the Fourier transform of $f$. The function class in question is compared with related classes already considered in the literature.
\end{abstract}

Keywords: Radial functions, Fourier transform, asymptotics, multipliers

AMS subject classification: $42 \mathrm{~B} 10$

\section{Introduction}

Let $\mathbb{R}^{n}$ be the $n$-dimensional Euclidean space with elements $x=\left(x_{1}, \ldots, x_{n}\right)$. If a function $f$ is integrable, in the Lebesgue sense, over all $\mathbb{R}^{n}$, there is no problem to understand its Fourier transform

$$
\hat{f}(x)=\int_{\mathbb{R}^{n}} f(u) e^{-i x \cdot u} d u
$$

where $x \cdot u=x_{1} u_{1}+\ldots+x_{n} u_{n}$ is the scalar product of $u, x \in \mathbb{R}^{n}$. The formal inverse formula

$$
f(u)=(2 \pi)^{-n} \int_{\mathbb{R}^{n}} \hat{f}(x) e^{i u \cdot x} d x
$$

for general $f \in L^{1}\left(\mathbb{R}^{n}\right)$ only holds in the sense that the integral on the right-hand side is summable to $f$ by some summability method. Under additional hypotheses, the Fourier transform of a non-integrable function may exist in a classical sense. For example, if $f$ is an integrable radial function, the $n$-dimensional Fourier integral reduces to the one-dimensional integral (see, e.g., [19: Chapter IV])

$$
\hat{f}(x)=\hat{f}_{0}(|x|)=(2 \pi)^{n / 2} \int_{0}^{\infty} f_{0}(t)(|x| t)^{-(n / 2-1)} J_{n / 2-1}(|x| t) t^{n-1} d t
$$

E. Liflyand: Bar-llan Univ., Dept. Math \& Comp. Sci., Ramat-Gan 52900, Israel; e-mail: liflyand@macs.biu.ac.il

W. Trebels: FB Math. Techn. Univ., Schloßgartenstr. 7, D - 64289 Darmstadt; e-mail: trebels@mathematik.tu-darmstadt.de

This paper was started during the first author's visit to the Technische Hochschule Darmstadt (THD) in 1995 under partial support of the Minerva Foundation in Germany through the Emmy Noether Institute at Bar-Ilan University and the THD. The paper was finished in 1996 at the THD in the framework of a DAAD Scholarship of the first author.

ISSN 02:32-2064 / \$2.50 (C) Heldermann Verlag Berlin 
where $|x|$ denotes the Euclidean norm of $x$ and $J_{\mu}$ is the Bessel function of first type and order $\mu$. This makes sense also for radial $L^{p}$-functions with $1<p<2 n /(n+1)$ : in that case $\hat{f}$ is everywhere continuous away from the origin. Another representation of the Fourier transform for radial, not necessarily integrable functions was obtained in $[15,16]$. It reads for $n=3$ as follows (for the general result, see Theorem $A$ below):

Let $n=3$ and $f$ be a radial function in $\mathbb{R}^{3}$ so that $f_{0}$ is bounded on $[0, \infty)$ and locally absolutely continuous on $(0, \infty)$. Suppose further that $\lim _{t \rightarrow \infty} f_{0}(t)=0$ and $\lim _{t \rightarrow \infty} t f_{0}^{\prime}(t)=0$, and $t f_{0}^{\prime}(t)$ is of bounded variation on $[0, \infty)$. Then

$$
\hat{f}(u)=-(2 \pi)^{3 / 2}|u|^{-1 / 2} \int_{0}^{\infty}\left[t f_{0}^{\prime}(t)\right] t^{3 / 2}\left[\int_{0}^{1} s^{3 / 2} J_{1 / 2}(|u| t s) d s\right] d t,
$$

and $\hat{f}$ coincides with the distributional Fourier transform of $f$; for $|x|>0$

$$
f(x)=\lim _{A \rightarrow \infty}(2 \pi)^{-3} \int_{|u| \leq A} \hat{f}(u) e^{i x \cdot u} d u .
$$

This also illustrates the fact that many results for radial functions are of one-dimensional nature. Our main result (see Theorem 1) is to be scen on this background: it reduces the Fourier transform of a radial function from some class not only to a one-dimensional integral but to the one-dimensional Fourier transform of some related function. In three dimensions, for functions $f$ as above, it reads as follows:

$$
\hat{f}(x)=4 \pi|u|^{-2} \int_{0}^{\infty}\left[t f^{\prime}(t)\right] \cos |u| t d t+\text { remainder terms } \quad\left(u \in \mathbb{R}^{3}\right) .
$$

The outline of this paper is as follows. First we introduce a class of functions already used in $[15,16]$ and compare it with some other classes (see $[10,21]$ ) in Section 2. In Section 3 we give our main result, the precise form of (1) and for arbitrary dimension. Applications, in particular to the multiplier problem, are indicated in Section 4; for instance, Example 2 contains the construction of some linear means $L_{N}^{\varphi}$ for multivariate Fourier series with $\left\|L_{N}^{\infty}\right\|=O(\ln \ln \ln N)$.

We use the same letter $C$ for different constants independent of substantial parameters.

\section{Classes of functions related to bounded variation}

For $0<\delta<1$ and a locally integrable function $g$ on $(0, \infty)$ define the fractional (Weyl type) integral of order $\delta$ by

$$
W_{\omega}^{\delta}(g)(t)= \begin{cases}\frac{1}{\Gamma(\delta)} \int_{t}^{\nu} g(r)(r-t)^{\delta-1} d r & \text { if } 0<t<\omega \\ 0 & \text { if } t \geq \omega\end{cases}
$$

and, following Cossar [8], a fractional Weyl derivative of order $\alpha$ by

$$
g^{(\alpha)}(t)=\lim _{\omega \rightarrow \infty}-\frac{d}{d t} W_{-\infty}^{\prime-\alpha}(g)(t)
$$


when $0<\alpha<1$ and

$$
g^{(\alpha)}(t)=\left(\frac{d}{d t}\right)^{p} g^{(\delta)}(t)
$$

when $\alpha=p+\delta$ with $p=1,2, \ldots$, and $0<\delta<1$.

Denote by $A C_{\mathrm{loc}}$ and $B V_{\mathrm{loc}}$ the classes of functions locally absolutely continuous and locally of bounded variation, respectively.

Let $\alpha^{*}$ be the greatest integer less than $\alpha$. Consider the class $M V_{\alpha+1}^{b}$, with $\alpha>0$ and $b \geq 0$, of $C(0, \infty)$-functions satisfying the following conditions (cf. $[15,16]$ ):

$$
\begin{aligned}
& g, g^{\prime}, \ldots, g^{\left(\alpha^{*}\right)} \quad \text { are locally absolutely continuous on }(0, \infty) . \\
& \lim _{t \rightarrow \infty} g(t)=0, \quad \lim _{t \rightarrow \infty} t^{\alpha+b} g^{(\alpha)}(t)=0 . \\
& \|g\|_{M V_{\alpha+1}^{b}}:=\sup _{t>0}\left|t^{b} g(t)\right|+\int_{0}^{\infty}\left|d\left(t^{\alpha+b} g^{(\alpha)}(t)\right)\right|<\infty .
\end{aligned}
$$

This space is related to the class $B V_{a+1}^{b}$ of $C(0, \infty)$-functions satisfying the following conditions (see, e.g., [7] or [23: p. 1181]):

$$
\begin{aligned}
& g^{(\alpha-[\alpha])}, \ldots, g^{(\alpha-1)} \in A C_{\mathrm{loc}}(0, \infty) . \\
& \lim _{t \rightarrow \infty} g(t)=0, \quad g^{(\alpha)} \in B V_{\mathrm{loc}}(0, \infty) . \\
& \|g\|_{B V_{o+1}^{b}}:=\int_{0}^{\infty} t^{\alpha+b}\left|d g^{(\alpha)}(t)\right|<\infty
\end{aligned}
$$

and the class $W B V_{\infty, \alpha+1}^{b}$, as considered, e.g., in $[10,11]$, of $C(0, \infty)$-functions satisfying (2') and additionally $g^{(\alpha)} \in A C_{\mathrm{loc}}(0, \infty)$, and

$$
\|g\|_{W B V_{\infty, a+1}^{0}}:=\underset{t>0}{\operatorname{esssup}}\left|t^{b} g(t)\right|+\underset{t>0}{\operatorname{esssup}}\left|t^{\alpha+1+b} g^{(\alpha+1)}(t)\right|<\infty .
$$

The following lemma establishes relations between these classes.

Lemma 1. In the sense of continuous embedding there holds

$$
B V_{\alpha+1}^{b} \hookrightarrow M V_{\alpha+1}^{b} \hookrightarrow W B V_{\infty, \alpha}^{b}
$$

None of these embeddings holds in the opposite direction.

Proof. We only discuss the instance $b=0$ which is the worst case. Here and in what follows we omit the superscript $b$ in the case $b=0$. Suppose $g \in B V_{\alpha+1}$. Then, by [23: Lemma 1.1],

$$
\int_{0}^{\infty}\left|d\left(t^{\alpha} g^{(\alpha)}(t)\right)\right| \leq C \int_{0}^{\infty} t^{(\alpha-1}\left|g^{(\alpha)}(t)\right| d t+C \int_{0}^{\infty} t^{\alpha}\left|d g^{(\alpha)}(t)\right| \leq C\|g\|_{\beta} v_{\alpha+1}
$$

showing the norm estimate for the left inclusion. Trivially, a function $h$ of bounded variation, vanishing at infinity, sattisfies $|h(t)| \leq \int_{0}^{\infty}|d h(t)|$, hence $\|g\|_{w} \beta v_{\infty, \ldots} \leq\|g\|_{M v_{1+1}}$. 
The absolute continuity properties follow from the discussion in [10: Section 3]. Further, by [22: Lemma 3.15],

$$
\left|t^{\alpha} g^{(\alpha)}(t)\right|=C t^{\alpha}\left|\int_{t}^{\infty} d g^{(\alpha)}(s)\right| \leq C \int_{t}^{\infty} s^{\alpha}\left|d g^{(\alpha)}(s)\right| \quad\left(g \in B V_{\alpha+1}\right)
$$

which, by hypothesis, tends to zero as $t \rightarrow \infty$, a.e., so the cmbeddings are proved.

That the inclusions are strict show the examples $(b=0)$

$$
g_{1}(t)=\chi(t) \sin (\ln \ln (1 / t)),
$$

where $\chi \in C^{\infty}[0, \infty)$ is a cut-off function which equals to 1 for $0 \leq t \leq 1 / 4$ and vanishes for $t \geq 1 / 2$. Then $g_{1}^{\prime}(t)=-\cos (\ln \ln (1 / t)) /(t \ln (1 / t))$ for $0<t<1 / 4$ and $g_{1}^{\prime}(t)=0$ for $t>1 / 2$, hence $t g_{1}^{\prime}(t)$ is of bounded variation on $[0, \infty)$ but

$$
\left\|g_{1}\right\|_{B V_{1}} \geq \int_{0}^{1 / 4}\left|g_{1}^{\prime}(t)\right| d t=\int_{\ln 4}^{\infty}|\cos (\ln u)| d u / u \geq \int_{1}^{\infty}|\cos v| d v=\infty,
$$

thus $g_{1} \notin B V_{2}$ since $\left\|g_{1}\right\|_{B V_{1}} \leq C\left\|g_{1}\right\|_{B V_{2}}$. This argument also works for all $\alpha>0$, $b=0$, if one replaces $g_{1}$ by $(\alpha=p+\delta, 0<\delta \leq 1)$

$$
g_{1, \alpha}(t)=\int_{t}^{1}(u-t)^{\delta-1} \frac{\cos (\ln \ln (1 / u))}{u^{\delta} \ln (1 / u)} \chi(u) d u .
$$

Note that $g_{1, \alpha}$ is bounded and that $g_{1, \alpha}^{(\delta)}(t)=C \cos (\ln \ln (1 / t))\left(t^{\delta} \ln (1 / t)\right)^{-1} \chi(t)$. In the case $\alpha=1$, E. Belinskii (see [15]) suggested to replace (5) by $g_{2}(t)=g_{1}(t) / \ln \ln (1 / t)$ which also yields an example for the strict embeddings; observe that $g_{2}$ is continuous at the origin and vanishes there in contrast to (5).

Similarly, consider $g_{3}(t)=t^{i \gamma}$, with $\gamma \in \mathbb{R}$. Then $g_{3} \in W B V_{\infty, \alpha}$ for all $\alpha \geq 0$ and obviously $t^{i \gamma} \notin M V_{\alpha+1}$ for all $\alpha>0$ and $\gamma \in \mathbb{R}$ fixed

Lemma 2. If $g \in M V_{\alpha+1}^{b}$, then it follows for $p=1,2, \ldots, \alpha^{*}$ that

$$
\left\|t^{b+p} g^{(p)}(t)\right\|_{\infty} \leq C\|g\|_{M v_{a+1}^{b}} \quad \text { and } \quad \lim _{t \rightarrow \infty} t^{p} g^{(p)}(t)=0 .
$$

Proof. The first assertion is clear on account of the embedding behavior of the $W B V$-spaces: $W B V_{\infty, \alpha}^{b} \hookrightarrow W B V_{\infty, \beta}^{b}$ for $0<\beta<\alpha$. From this also the second assertion is obvious in the case $b>0$. So let $b=0$ and first suppose $\alpha$ is integer. Then the argument in [24: p. 193] shows the assertion in the case $\alpha=2$. That this argument also works for all $\alpha$ integer we illustrate at the case $\alpha=3$. Thus we show: $\lim _{t \rightarrow \infty} g(t)=0$ and $\lim _{\iota \rightarrow \infty} t^{3} g^{\prime \prime \prime}(t)=0$ implies $\lim _{t \rightarrow \infty} t^{2} g^{\prime \prime}(t)=0$ whence follows $\lim _{t \rightarrow \infty} t g^{\prime}(t)=0$ by the case $\alpha=2$. Let $0<\delta<1 / 4$ be arbitrary, fixed, $t>0$ be large. Then by Taylor's formula (at $s=0$ ).

$$
\begin{aligned}
G(s):=\Delta_{s}^{2} g(t) & =g(t+2 s)-2 g(t+s)+g(t) \\
& =g^{\prime \prime}(t) s^{2}+\left(s^{3} / 3 !\right)\left(8 g^{\prime \prime \prime}(t+2 \theta s)-2 g^{\prime \prime \prime}(t+\theta s)\right)
\end{aligned}
$$


with $0<\theta<1$. Now choose $s= \pm \delta t$ and $T_{\delta}$ so large that $\left|\Delta_{\delta t}^{2} g(t)\right| \leq \delta^{3}$ for all $t \geq T_{\delta}$. Furthermore, observe that by hypothesis

$$
\left|G^{\prime \prime \prime}(\theta s)\right| \leq 8 M(t+2 \theta \delta t)^{-3}+2 M(t+\theta \delta t)^{-3} \leq 10 M t^{-3}(1-2 \delta)^{-3}
$$

and thus

$$
\left|t^{2} g^{\prime \prime}(t)\right|=\left|\delta^{-2} \Delta_{ \pm \delta \ell}^{2} g(t)-\delta t^{3} G^{\prime \prime \prime}(\theta s) / 3 !\right| \leq \delta\left(1+10 M(1-2 \delta)^{-3} / 3 !\right)
$$

for $t \geq T_{\delta}$, i.e., the assertion for $\alpha=3$. It is clear that by using higher differences, the remaining case of $\alpha$ integer can be proved by induction.

If $\alpha$ is fractional and $\alpha>1$, then we have, by Lernma 1, that $g \in W B V_{\infty,[\alpha]} \hookrightarrow$ $W B V_{\infty, j}, j=1, \ldots,[\alpha]$, and the argument for $\alpha$ integer shows $\lim _{t \rightarrow \infty} t^{j} g^{(j)}(t)=0$ for $j=1, \ldots,[\alpha]-1$. Thus we only have to look at the case $j=[\alpha]<\alpha$. By Lemma 1 , we have $g \in W B V_{\infty, \alpha}$ and hence the fractional calculus within the $W B V$-spaces (see [10, 11) may be applied to give with an integration by parts

$$
\begin{aligned}
g^{([\alpha])}(t)= & C \int_{t}^{\infty}(s-t)^{\alpha-[\alpha]-1} s^{-\alpha} s^{\alpha} g^{(\alpha)}(s) d s \\
= & \left.C\left(\int_{s}^{\infty}(\sigma-t)^{\alpha-[\alpha]-1} \sigma^{-\alpha} d \sigma\right) s^{\alpha} g^{(\alpha)}(s)\right|_{s=t} ^{\infty} \\
& +C \int_{\mathfrak{t}}^{\infty}\left(\int_{s}^{\infty}(\sigma-t)^{\alpha-[\alpha]-1} \sigma^{-\alpha} d \sigma\right) d\left(s^{\alpha} g^{(\alpha)}(s)\right) \\
= & I_{t}+I I_{t} .
\end{aligned}
$$

With the substitution $\sigma=t r$ the term $I_{t}$ can be estimated by $(s \geq t, \alpha>1)$

$$
\begin{aligned}
\left|t^{[\alpha]} I_{t}\right| & \leq\left. C\left(\int_{s / t}^{\infty}(r-1)^{\alpha-[\alpha]-1} r^{-\alpha} d r\right) s^{\alpha} g^{(\alpha)}(s)\right|_{s=t} ^{\infty} \\
& =C\left(\int_{1}^{\infty}(r-1)^{\alpha-[\alpha]-1} r^{-\alpha} d r\right) t^{\alpha} g^{(\alpha)}(t)
\end{aligned}
$$

thus $\left|t^{[\alpha]} I_{t}\right| \rightarrow 0$ as $t \rightarrow \infty$ since by hypothesis $\lim _{t \rightarrow \infty} t^{\alpha} g^{(\alpha)}(t)=0$.

Concerning $I I_{t}$ an interchange of integration gives

$$
\begin{aligned}
\left|I I_{t}\right| & =C \int_{t}^{\infty}(\sigma-t)^{\alpha-[\alpha]-1} \sigma^{-\alpha} \int_{t}^{\sigma}\left|d\left(s^{\alpha} g^{(\alpha)}(s)\right)\right| d \sigma \\
& \leq C t^{-[\alpha]} \int_{t}^{\infty}\left|d\left(s^{\alpha} g^{(\alpha)}(s)\right)\right| \int_{1}^{\infty}(r-1)^{\alpha-[\alpha]-1} r^{-\alpha} d r
\end{aligned}
$$

thus also $\left|t^{\{\alpha\}} I I_{t}\right| \rightarrow 0$ as $t \rightarrow \infty$ since $\int_{0}^{\infty}\left|d\left(s^{\alpha} g^{(\alpha)}(s)\right)\right|<\infty$. Combining these two situations gives the remaining case $\lim _{t \rightarrow \infty} t^{[\alpha]} g^{([\alpha])}(t)=0$ 


\section{A class of radial functions and the Fourier transform}

Now we are able to describe the passage from the multidimensional Fourier transform to the one-dimensional Fourier transform of a related function.

Theorem 1. Let $f_{0} \in M V_{\alpha+1}^{b}$ with $0<\alpha \leq(n-1) / 2$ and $b=(n-1) / 2-\alpha$, set $F_{\alpha}(t)=t^{(n-1) / 2} f_{0}^{(\alpha)}(t)$. Then there holds, for the radial extension $f(x)=f_{0}(|x|)$ of $f_{0}$,

$$
\begin{aligned}
\hat{f}(x)=|x|^{-(n-1) / 2-\alpha}\{ & C_{1, n} \int_{0}^{\infty} F_{\alpha}(t) \cos (|x| t-\pi(n+2 \alpha-1) / 4) d t \\
& +C_{2, n}|x|^{-1} F_{\alpha}(\pi /(2|x|)) \\
& \left.+O\left(|x|^{-1} \int_{0}^{\infty} \min (2|x| s / \pi, \pi /(2|x| s))\left|d F_{\alpha}(s)\right|\right)\right\}
\end{aligned}
$$

where $C_{1, n}=2^{(n+1) / 2} \pi^{(n-1) / 2}(-1)^{\alpha^{*}+1}$ and $C_{2, n}$ is a constant depending only on $n$ and $\alpha$, given in (8) below.

Remark 1. In $[15,16]$ a weaker form of Theorem 1 was derived in the case $\alpha=$ $(n-1) / 2$ (hence $b=0$ ). Unfortunately this weaker form was not applicable to generalize results from [14].

Remark 2. Concerning the terms in the brackets on the right-hand side of (6), the first one is the clained one-dimensional Fourier transform for which in a concrete situation an explicit formula may exist - there are numerous detailed tables (sce, e.g., [2]). The integral exists as an improper Riemann integral since $F_{\alpha} \in B V[0, \infty)$ and varnishes at infinity.

The second term in the brackets, being given explicitly, is easy to handle. It may

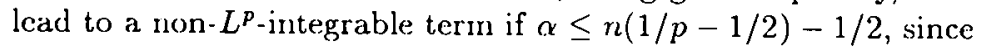

$$
\int_{1}^{N} r^{n-1}\left|r^{-(n-1) / 2-\alpha-1} F_{\alpha}(\pi /(2 r))\right|^{p} d r
$$

is in any case uniformly bounded in $N$ if $\alpha>n(1 / p-1 / 2)-1 / 2$.

The third one is trivially dominated by $|x|^{-1}|| f_{0} \|_{M v_{o+1}^{b}}$ and thus may be interpreted as a remainder term. It may lead to a non- $L^{p}$-integrable term if (in difference to the second term) $\alpha<n(1 / p-1 / 2)-1 / 2$, since

$$
\int_{1}^{N} r^{n-1}\left|r^{-(n-1) / 2-\alpha-1} \int_{0}^{\infty} \min ((2 r s) / \pi, \pi /(2 r s))\right| d F_{c r}(s)||^{p} d r
$$

is uniformly bounded in $N$ provided $\alpha \geq n(1 / p-1 / 2)-1 / 2$ (the case $p=1, \alpha=(n-1) / 2$ was already considered in $[15,16]$; see also $[3,4])$.

Remark 3. For radial functions with compact support and integrable Fourier transform, A. Podkorytov [17] obt,ained a similar formula. Under very restrietive assumptions, in [18: Chapter 5/Lomma 25.1'] the multidinensional Fourier transform of a radial function is reduced to the onc-dimensional onc. result:

Remark 4. In the proof of Theorem 1 there will be essentially used the following 
Theorem A. Let $f_{0}$ be a function in $M V_{a+1}^{b}$ and set.

$$
Q_{\alpha}(t)=\int_{0}^{1}(1-s)^{\alpha-1} s^{n / 2} J_{n / 2-1}(t s) d s
$$

Then

$$
\hat{f}(u)=\frac{(2 \pi)^{n / 2}(-1)^{\alpha^{*}+1}}{\Gamma(\alpha)}|u|^{1-n / 2} \int_{0}^{\infty} F_{\alpha}(t) t^{\alpha+1 / 2} Q_{\alpha}(|u| t) d t
$$

is continuous, tends to zero as $|u| \rightarrow \infty$, and coincides with the distributional Fourier transform of $f$; for $|x|>0$ the following inverse formula holds:

$$
f(x)=\lim _{A \rightarrow \infty}(2 \pi)^{-n} \int_{|u| \leq A}\left(1-|u|^{2} / A^{2}\right)^{(n-1) / 2-\alpha} \hat{f}(u) e^{i x \cdot u} \cdot d u .
$$

Both integrals converge uniformly away from the origin.

In $[15,16]$ this result was obtained under some additional conditions at the origin and infinity which are removed by Lemma 2 . We note that Theorem $A$ can handle radial functions which are not $L^{p}$-integrable, $p<\infty$; e.g., $f_{0}=\ln ^{-1}(e+t), \alpha=(n-1) / 2$. Further observe that the restriction $\alpha \leq(n-1) / 2$ is somehow natural, since $f \in L_{\mathrm{rad}}^{1}$ yields $\hat{f}_{0} \in B V_{\gamma+1}$ with $\gamma<(n-3) / 2$. Representations similar to (7) with $\alpha>$ $n(1 / p-1 / 2)-1 / 2$ have been used earlier (see, e.g., [23]), where one has the absolute convergence of the integrals involved. Theorem $\mathrm{A}$ is mainly based on the handling of improper Riemann integrals.

\section{Proof of Theorem 1. Write}

$$
\Phi_{\alpha}(r)=(1 / \Gamma(\alpha)) r^{\alpha+1 / 2} Q_{\alpha}(r)-\sqrt{2 / \pi} \cos (r-\pi(n+2 \alpha-1) / 4)
$$

Then (7) may be written in the form

$$
\begin{aligned}
\hat{f}(x)= & C_{1, n}|x|^{-(n-1) / 2-\alpha} \int_{0}^{\infty} F_{\alpha}(t) \cos (|x| t-\pi(n+2 \alpha-1) / 4) d t \\
& +(2 \pi)^{n / 2}(-1)^{\alpha^{*}+1}|x|^{-(n-1) / 2-\alpha} \int_{0}^{\infty} F_{\alpha}(t) \Phi_{\alpha}(|x| t) d t
\end{aligned}
$$

and only the last term has to be discussed. Decompose it into

$$
F_{\alpha}(\pi /(2|x|)) \int_{0}^{\infty} \Phi_{\alpha}(|x| t) d t+\int_{0}^{\infty}\left[F_{\alpha}(t)-F_{\alpha}(\pi /(2|x|))\right] \Phi_{\alpha}(|x| t) d t=I_{1}+I_{2}
$$

We have

$$
\begin{aligned}
I_{1} & =|x|^{-1} F_{\alpha}(\pi /(2|x|)) \int_{0}^{\infty} \Phi_{\alpha}(t) d t \\
& =C_{2, n}(2 \pi)^{-n / 2}(-1)^{\alpha^{*}+1}|x|^{-1} F_{\alpha}(\pi /(2|x|))
\end{aligned}
$$


provided $\int_{0}^{\infty} \Phi_{\alpha}(t) d t$ is finite. The integral is obviously convergent over $[0,1]$. Thus it remains to estimate $\Phi_{\alpha}(t)$ on $[1, \infty)$. We need the following relation (see [15: Lemma 2]): For $r \geq 1, \beta>-1 / 2, \mu>-1$ and each positive integer $p$

$$
\int_{0}^{1}(1-s)^{\mu} s^{\beta+1} J_{\beta}(r s) d s=\sum_{j=1}^{p} \alpha_{j}^{\mu} r^{-(\mu+j)} J_{\beta+\mu+j}(r)+O\left(r^{-\mu-p-3 / 2}\right)
$$

where $\alpha_{j}^{\mu}$ are some numbers depending only on $p$ and $\mu$, and $\alpha_{1}^{\mu}=\Gamma(\mu+1), \alpha_{2}^{\mu}=$ $\mu \Gamma(\mu+2)$. By (9) we have

$$
\begin{aligned}
Q_{\alpha}(t) & =\int_{0}^{1}(1-s)^{\alpha-1} s^{n / 2} J_{n / 2-1}(t s) d s \\
& =\Gamma(\alpha) t^{-\alpha} J_{n / 2+\alpha-1}(t)+(\alpha-1) \Gamma(\alpha+1) t^{-\alpha-1} J_{n / 2+\alpha}(t)+O\left(t^{-\alpha-5 / 2}\right) .
\end{aligned}
$$

Using also the asymptotic formula for the Bessel function (see, e.g., $[1: \S 7.13 .1(3)]$ )

$$
\begin{aligned}
J_{\nu}(t)= & \sqrt{2 /(\pi t)} \cos (t-\pi \nu / 2-\pi / 4) \\
& +\sqrt{2 / \pi}\left[\left(1-4 \nu^{2}\right) / 8\right] t^{-3 / 2} \sin (t-\pi \nu / 2-\pi / 4)+O\left(t^{-5 / 2}\right)
\end{aligned}
$$

as $t \rightarrow \infty$, we obtain

$$
\begin{aligned}
\Phi_{\alpha}(t)= & 2^{-5 / 2} \pi^{-1 / 2}\left[1-4(n / 2+\alpha-1)^{2}\right] t^{-1} \sin (t-\pi(n+2 \alpha-1) / 4) \\
& +\frac{(\alpha-1) \Gamma(\alpha+1)}{\Gamma(\alpha)} \sqrt{2 / \pi} t^{-1} \cos (t-\pi(n+2 \alpha-1) / 4)+O\left(t^{-2}\right)
\end{aligned}
$$

and the integral in question is convergent.

Split $I_{2}$ into two parts. We have

$$
\begin{aligned}
\int_{0}^{\pi /(2|x|)} & {\left[F_{\alpha}(t)-F_{\alpha}(\pi /(2|x|))\right] \Phi_{\alpha}(|x| t) d t } \\
= & -\int_{0}^{\pi /(2|x|)} \Phi_{\alpha}(|x| t) d t \int_{t}^{\pi /(2|x|)} d F_{\alpha}(s) \\
= & -\int_{0}^{\pi /(2|x|)} d F_{\alpha}(s) \int_{0}^{s} \Phi_{\alpha}(|x| t) d t \\
= & O\left(\int_{0}^{\pi /(2|x|)}\left|d F_{\alpha}(s)\right| \int_{0}^{s}\left[(|x| t)^{\alpha+1 / 2}(|x| t)^{n / 2-1}+1\right] d t\right) \\
= & O\left(\int_{0}^{\pi /(2|x|)} s\left|d F_{\alpha}(s)\right|\right) \\
= & |x|^{-1} O\left(\int_{0}^{\pi /(2|x|)}|x| s\left|d F_{\alpha}(s)\right|\right) .
\end{aligned}
$$


Further,

$$
\begin{aligned}
& \int_{\pi /(2|x|)}^{\infty}\left[F_{\alpha}(t)-F_{\alpha}(\pi /(2|x|))\right] \Phi_{\alpha}(|x| t) d t \\
&=\int_{\pi /(2|x|)}^{\infty} \Phi_{\alpha}(|x| t) d t \int_{\pi /(2|x|)}^{t} d F_{\alpha}(s) \\
&=\int_{\pi /(2|x|)}^{\infty} d F_{\alpha}(s) \int_{s}^{\infty} \Phi_{\alpha}(|x| t) d t \\
&=|x|^{-1} \int_{\pi /(2|x|)}^{\infty} d F_{\alpha}(s) \int_{|x| s}^{\infty} \Phi_{\alpha}(t) d t
\end{aligned}
$$

and finally, by the above asymptotic for $\Phi_{\alpha}$,

$$
\begin{aligned}
\int_{|x| s}^{\infty} \Phi_{\alpha}(t) d t= & 2^{-5 / 2} \pi^{-1 / 2}\left[1-4(n / 2+\alpha-1)^{2}\right] \\
& \times \int_{|x| s}^{\infty} \sin (t-\pi(n+2 \alpha-1) / 4) d t / t \\
& +\frac{(\alpha-1) \Gamma(\alpha+1)}{\Gamma(\alpha)} \sqrt{2 / \pi} \int_{|x| s}^{\infty} \cos (t-\pi(n+2 \alpha-1) / 4) d t / t \\
& +O\left(\int_{|x| s}^{\infty} t^{-2} d t\right)=O\left((|x| s)^{-1}\right)
\end{aligned}
$$

This proves the theorem

\section{Applications}

Let us generalize some recent one-dimensional results in [14] to the radial case. For $1<q<\infty$ set

$$
\|g\|_{A_{q}}=\int_{0}^{\infty}\left(\frac{1}{u} \int_{u \leq|\ell| \leq 2 u}|g(t)|^{q} d t\right)^{1 / q} d u
$$

while for $q=\infty$

$$
\|g\|_{A_{\infty}}=\int_{0}^{\infty} \operatorname{ess} \sup _{u \leq|t| \leq 2 u}|g(t)| d u
$$

for the behavior of the Fourier transform on these spaces, see also [12, 21]. Let us define $H_{B T}$ as the space of integrable functions satisfying

$$
\|g\|_{H_{B T}}=\int_{\mathbb{R}}|g(t)| d t+\int_{0}^{\infty}\left|\int_{|t| \leq s / 2} \frac{g(s-t)-g(s+t)}{t} d t\right| d s<\infty
$$

which is an integral version of the series condition due to Boas-Telyakovskii. For this and relations to the Hilbert transform and the real Hardy space $R e H^{1}$, see [14]. Although the spaces $A^{p}$ are not embedded in $R e H^{1}$ the following embeddings hold:

$$
A_{\infty} \hookrightarrow A_{p_{1}} \hookrightarrow A_{p_{2}} \hookrightarrow H_{B T} \hookrightarrow L^{1} \quad\left(p_{1}>p_{2}>1\right)
$$

The main result of the paper [14] may be slightly reformulated in the following form: 
Theorem B. Let $\lambda$ be a locally absolutely continuous function with $\lim _{|t| \rightarrow \infty} \lambda(t)=$ 0 and $\lambda^{\prime} \in H_{B T}$. Then

$$
\hat{\lambda}(r)=i r^{-1}(\lambda(\pi /(2|r|))-\lambda(-\pi /(2|r|)))+\theta \gamma(r) \quad(|r|>0)
$$

where $|\theta| \leq C$ and $\int_{\mathbb{R}}|\gamma(r)| d r \leq\left\|\lambda^{\prime}\right\|_{H_{B T}}$.

Among various applications of this result and the like (see, e.g., [12, 14]), we point at a somewhat unexpected one, namely to problems of best approximation of infinitely differentiable functions by entire functions (see [9]).

We are now able to generalize Theorem $B$ to the radial case. Since Theorem B is essentially an $L^{1}$-theorem we restrict ourselves to the case $\alpha=(n-1) / 2$. For the sake of simplicity, let us use the notation $F(t):=F_{(n-1) / 2}(t)=t^{(n-1) / 2} f_{0}^{((n-1) / 2)}(t)$ in what follows.

Theorem 2. Let $f_{0} \in M V_{(n+1) / 2}$; assume additionally $F$ to be locally absolutely continuous and $F^{\prime} \in H_{B T}$. Then

$$
\hat{f}(x)=|x|^{-n}\left[C_{1, n} \sin (\pi(n-1) / 2)+C_{2, n}\right] F(\pi /(2|x|))+\theta|x|^{1-n} \gamma(|x|) \quad(|x|>0)
$$

where $C_{1, n}$ and $C_{2, n}$ are as in Theorem 1 while $\theta$ and $\gamma$ as in Theorem $B$.

Proof. First of all the formula from Theorem B may be rewritten for functions defined on the half-axis $(0, \infty)$ as follows:

$$
\int_{0}^{\infty} \lambda(t) \cos (r t+\mu) d t=r^{-1} \sin \mu \lambda(\pi /(2|r|))+\theta \gamma(r)
$$

Now this and (6) proves Theorem 2. One has only to recall that in (6) the third term is integrable for $\alpha=(n-1) / 2$ (see Remark 2)

Let us apply Theorem 2.

Example 1. Let $n=3$ and $f=g_{1}$ be given by (5). Observe that $F(t)=t f^{\prime}(t) \epsilon$ $A_{q}, q>1$, and $\|\gamma\|_{L^{1}(\mathbb{R})} \leq\left\|F^{\prime}\right\|_{H_{B T}} \leq C_{q}\left\|F^{\prime}\right\|_{A_{q}}$, thus, by (10), the hypotheses of Theorem 2 are satisfied. Hence

$$
\hat{f}(x)=C|x|^{-3} \ln ^{-1}(2 e|x| / \pi) \cos (\ln \ln (2 e|x| / \pi))+\theta|x|^{-2} \gamma(|x|) \quad\left(|x|>\pi / 2, x \in \mathbb{R}^{3}\right)
$$

where $|\theta| \leq C$ and $\int_{\pi / 2}^{\infty}|\gamma(t)| d t<\infty$. This is indeed an asymptotic formula since the second term on the right-hand side is integrable outside the ball with the radius $\pi / 2$ unlike the first term which is not integrable! This can be extended at once to the other dimensions when using (5') with $\delta=1 / 2$.

Example 2. Let us consider the following linear means of the Fourier series of $2 \pi$-periodic in each variable functions $f \in L^{1}\left(\mathbb{T}^{n}\right)$ :

$$
L_{N}^{\varphi}(f ; x)=\sum_{k \in \mathbf{Z}^{n}} \varphi(k / N) \hat{f}(k) e^{i k \cdot \boldsymbol{x}}, \quad \hat{f}(k)=\frac{1}{(2 \pi)^{n}} \int_{\mathbb{T}^{n}} f(x) e^{-i k \cdot x} d x .
$$

The following theorem is known for the $L^{1}$-norms of these means. 
Theorem C. Let $\varphi(x)=\varphi_{0}(|x|)$ be a radial function such that $\varphi_{0} \in M V_{(n+1) / 2}$ and continuous at the origin. Then

$$
\left\|L_{N}^{\varphi}\right\|=\frac{1}{(2 \pi)^{n}} \int_{|x| \leq \pi N}|\hat{\varphi}(x)| d x+O\left(\|\Phi\|_{B V(0, \infty)}\right)
$$

where $\Phi(t)=t^{(n-1) / 2} \varphi_{0}^{((n-1) / 2)}(t)$.

Actually Theorem $\mathrm{C}$ is given (see $[3,4]$ ) under some additional assumptions on $\varphi$ which are removed by Lemma 2 (cf. Theorem $\mathrm{A}$ and below). Let $n=3$ and $\varphi_{0}(t)=$ $\left(1-g_{2}(t)\right) \chi(t)$, where $\chi$ and $g_{2}$ are described below (5'). Note that we have $\varphi_{0}(0)=1$ which is necessary for approximation. If we apply Theorem $\mathrm{C}$ and then Theorem 2, we obtain

$$
\begin{aligned}
\left\|L_{N}^{\varphi}\right\| & =C \int_{1 \leq|x| \leq \pi N} \frac{|\cos (\ln \ln (2|x| / \pi))|}{|x|^{n} \ln (2|x| / \pi) \ln \ln (2|x| / \pi)} d x+O(1) \\
& =C \int_{1}^{N} \frac{|\cos (\ln \ln r)|}{r \ln r \ln \ln r} d r+\dot{O}^{\prime}(1) \\
& =C \ln \ln \ln N+O(1) .
\end{aligned}
$$

Replacing $\ln \ln (1 / t)$ by a "longer" In-chain in the denominator of the function $g_{2}$ one can get a worse behavior in $N$ of $\left\|L_{N}^{\varphi}\right\|$.

Remark 5. To derive Theorem 2, a weaker version of Theorem 1 (see $[15,16]$ ) mentioned above is not applicable since it assumes that $\int_{0}^{1}|F(t)| d t / t<\infty$. Note, however, that this weaker version is sufficient to generalize the Zygmund-Bochkarev theorem (see $[5,6])$ to the radial case (see $[3,15]$ ).

Remark 6. A result similar to Theorem 2 was proved earlier for boundedly supported radial functions with convex $F$ (see $[3,16]$, in this case convexity implies $\int_{0}^{1}|F(t)|$ $d t / t<\infty$; for precursory results, see $[13,20\})$.

For functions with compact support there arises the question, what is the impact of the behavior of the function near the right endpoint of the support. This will be considered in details in a paper to appear.

\section{References}

[1] Bateman, G. and A. Erdélyi: Higher Transcendental Functions. Volume II. New York: McGraw Hill Book Comp. 1953.

[2] Bateman, G. and A. Erdélyi: Tables of Integral Transforms. Volume I. New York: McGraw Hill Book Comp. 1954.

[3] Belinsky, E. S. and E. R. Liflyand: Lebesgue constants and integrability of the Fourier transform of a radial function (in Russian). Dokl. Acad. Sci. Ukraine (1980)6, 5 - 10.

[4] Belinsky, E. S. and E. R. Liflyand: On asymptotic behavior of Lebesgue constants of radial summability methods, (in Russian). In: Constructive Theory and Functions and Theory 
of Mappings (ed.: G. Suvorov, V. Belyi and V. Gutlyanskii). Kiev: Nauk. dumka 1981, pp. $49-77$.

[5] Bochkarev, S. V.: On a problem of Zygmund (in Russian). Izv. Akad. Nauk SSSR, Ser. Mat. 37 (1973), 630 - 638; Engl. transl. in Math. USSR Izvestija 37 (1973), 629 - 637.

[6] Bochkarev, S. V.: A Method of Averaging in the Theory of Orthogonal Series and Some Problems in the Theory of Bases (in Russian). Trudy Inst. Steklova 146 (1978); Engl. transl. Proc. Steklov Inst. Math. (1980)3, vi +92 pp.

[7] Butzer, P. L, Nessel, R. J. and W. Trebels: On radial $M_{p}^{q}$ Fourier multipliers. In: Math. Struct., Comput. Math., Math. Modelling (ed.: B. Sendov). Sofia: Publ. House Bulg. Acad. Sci. 1975, pp. $187-193$.

[8] Cossar, J.: A theorem on Cesàro surnmability. J. London Math. Soc. 16 (1941), 56 - 68.

[9] Ganzburg, M. and E. Liflyand: Estimates of best approximation and Fourier transforms in integral metrics. J. A ppr. Theory 83 (1995), $347-370$.

[10] Gasper, G. and W. Trebels: A characterization of localized Bessel potential spaces and applications to Jacobi and Hankel multipliers. Studia Math. 65 (1979), $243-278$.

[11] Gasper, G. and W. Trebels: Multiplier criterio of Hörmander type for Fourier series and applications to Jacobi series and Hankel transforms. Math. Ann. 242 (1979), $225-240$.

[12] Giang, D. V. and F. Moricz: Multipliers of Fourier transforms and series on $L^{1}$. Arch. Math. 62 (1994), 230 - 238.

[13] Liflyand, E. R.: On some conditions of integrability of Fourier transform (in Russian). Ukranian Math. J. 32 (1980), 110 - 118.

[14] Liflyand, E. R.: Asymptotics of Fourier transform for certain classes of functions. Anal. Math. 19 (1993), 151 - 169.

[15] Liflyand, E. R.: Fourier transforms of radial functions. Int. Transf. Spec. Funct. 4 (1996), $279-300$.

[16] Liflyand, E. R.: Some Conditions for Existence and Integrability of the Fourier Transform. Preprint 1995, 33 pp.

[17] Podkorytov, A. N.: Linear means of spherical Fourier sums (in Russian). In: Operator 'Theory and Function Theory, Volume 1 (ed.: M. Z. Solomyak). Leningrad: State Univ. 1983, pp. $171-177$.

[18] Samko, S. G., Kilbas, A. A. and O. I. Marichev: Fractional Integrals and Derivatives. Theory and Applications. New York: Gordon \& Breach Sci. Publ. 1992.

[19] Stein, E. M. and G. Weiss: Introduction to Fourier Analysis on Euclidean Spaces. Princeton (New Jersey): Univ. Press 1971.

[20] Trigub, R. M.: Conditions and asymptotic behavior of the Fourier transform of a radial function (in Russian). In: Metric Questions of Theory of Functions and Mappings (ed.: G. Suvorov and V. Belyi). Kiev: Nauk. dumka 1977, pp. $142-163$.

[21] Trigub, R. M.: Multipliers of Fourier series and approximation of functions by polynomials in spaces $C$ and $L$ (in Russian): Dokl. Akad. Nauk SSSR 306 (1989), 292 - 296; Engl. transl. in Soviet Math. Dokl. 39 (1989), $494-498$.

[22] Trebels, W.: Multipliers for $(C, \alpha)$-Bounded Fourier Expansions in Banach Spaces and Approximation Theory. Lect. Notes Math. 329 (1973), vi $+103 \mathrm{pp}$.

[23] Trebels, W.: Some Fourier multiplier criteria and the spherical Bochner-Riesz kernel. Rev. Roum. Math. Pures et Appl. 20 (1975), 1173 - 1185.

[24] Widder, D. V.: The Laplace Transform. Princeton (New Jersey): Univ. Press 1946. 